\title{
Ensemble approach combining multiple methods improves human transcription start site prediction
}

\author{
David G Dineen ${ }^{1,2^{*}}$, Markus Schröder ${ }^{3}$, Desmond G Higgins², Pádraig Cunningham ${ }^{1}$
}

\begin{abstract}
Background: The computational prediction of transcription start sites is an important unsolved problem. Some recent progress has been made, but many promoters, particularly those not associated with CpG islands, are still difficult to locate using current methods. These methods use different features and training sets, along with a variety of machine learning techniques and result in different prediction sets.

Results: We demonstrate the heterogeneity of current prediction sets, and take advantage of this heterogeneity to construct a two-level classifier ('Profisi Ensemble') using predictions from 7 programs, along with 2 other data sources. Support vector machines using 'full' and 'reduced' data sets are combined in an either/or approach. We achieve a $14 \%$ increase in performance over the current state-of-the-art, as benchmarked by a third-party tool.
\end{abstract}

Conclusions: Supervised learning methods are a useful way to combine predictions from diverse sources.

\section{Background}

The field of in-silico promoter prediction has developed greatly in recent years. Machine learning techniques, such as support vector machines and self-organising maps, and new features, especially those associated with structural properties of the DNA molecule, have led to progressive improvements in accuracy. The realization that the majority of the genome is transcribed [1-3], and that most promoters have diffuse clusters of multiple transcription start sites (TSS) [4], has led to a move away from discrete predictions, and towards scores for all base pairs of the genome. There is greater consensus on the correct way to evaluate predictions, reducing the biases inherent in the plethora of methods previously used [5].

Despite these developments, there is considerable need for improvement in promoter prediction performance. A bimodal distribution of $\mathrm{CpG}$ content splits human promoters into high and low-CpG content promoters [6]. Promoters with lower $\mathrm{CpG}$ content are associated with tissue-specific regulation [7], and are considered more

\footnotetext{
* Correspondence: david.dineen@ucd.ie

'Complex and Adaptive Systems Laboratory (CASL), University College

Dublin, Belfield, Dublin 4, Ireland

Full list of author information is available at the end of the article
}

difficult to predict [8]. Figure 1 shows histograms of scores for ARTS [9] and Profisi [10], two state-of-the-art methods. Many valid promoters receive low scores, and setting thresholds low enough to recover them will inevitably return many false positives.

One obvious way of improving performance is to combine several existing methods using an ensemble learning approach. Ensembles combining results from multiple programs have seen some use in promoter prediction $[11,12]$. They have also been successfully used in several other computational biology problem areas [13-15]. High diversity in individual methods is considered predictive of good ensemble accuracy [16]. It can be difficult, however, to improve on the performance of the best individual method [17]. In this paper, our aim was to explore whether the set of prediction methods was indeed diverse, and to improve predictive performance across the genome and at all thresholds.

Table 1 shows our chosen features. Most of the features are programs which were drawn from the top performers in a number of promoter prediction reviews [5,18]. It includes our own Profisi method [10], which we have previously shown to be very competitive. These programs are diverse in both features used and in machine learning methods. In addition, we included 


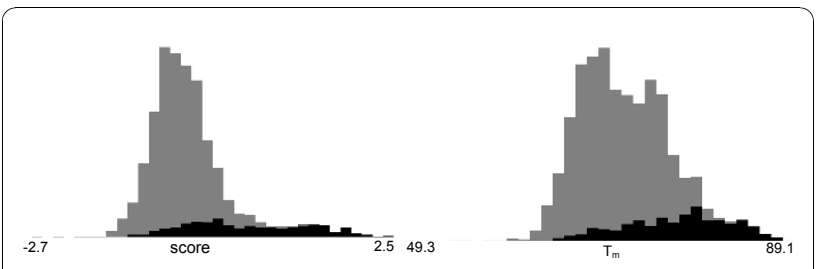

Figure 1 Promoter histogram scores. Histograms of scores from (a) ARTS, and (b) Profisi on 519 dbTSS promoters and 2,595 nonpromoters drawn from the ENCODE regions. ARTS scores are the output of an SVM, while Profisi scores are DNA melting

temperatures. Promoters are black and non-promoters are grey.

Specificity is high at high thresholds, but many promoters are given

low scores, and are obscured by non-promoters.

methylation profiles [19] and conservation scores from phylogenetic comparisons. Profisi is based on the observation that promoters are associated with high DNA melting temperature. DNA methylation both lowers melting temperature [20] and blocks promoter activity [21], but is not accounted for in the model used by Profisi. Hence, including methylation data could boost Profisi's performance. High conservation scores are considered predictive of functional areas of DNA [22].

As not all of our features are prediction scores, we did not use an averaging or voting-based ensemble method. Instead we used a support vector machine for aggregation. This also gave us the opportunity to use a non-linear kernel to increase separability of promoter and nonpromoter classes.

MetaProm [11] and EnsemPro [12] are both programs that use ensemble methods for promoter prediction. Although we were unable to obtain predictions for these programs, we could evaluate Profisi Ensemble using the evaluation rules described in the original papers in an attempt to make some comparison with them. MetaProm is based on an artificial neural network, and makes predictions in an area covering around $30 \%$ of the human genome, using a combination of dbTSS and RefSeq as the reference set. Multiple methods are discussed in the EnsemPro paper, but the most successful is weighted majority voting. It restricts its predictions to an area 1,150 base pairs either side of 400 TSS drawn from the Eukaryotic Promoter Database (EPD). The EPD is known to be strongly biased towards TATA box-containing promoters, which only comprise a small fraction of human promoters as a whole [23].

\section{Results and Discussion}

Table 2 shows the overlap between sets of predictions from seven popular promoter prediction programs, based on whole genome predictions for assembly hg17 of the human genome. Both true and false positives were counted, as variation in both will improve ensemble performance. Predictions from only two pairs of programs overlapped by $50 \%$ or more. The highest overlap was between ProSOM [24] and EP3 [25], which are by the same authors and use the same features. FirstEF [26], Eponine [27], and N-SCAN [28] also had reasonable overlap between prediction sets. The average overlap between pairs of predictions was $31.6 \%$. The low average overlap suggested that an ensemble approach was worth pursuing, as the ensemble would have good diversity. We further analysed overlap by splitting predictions into true (Table 3) and false (Table 4) positives. There was more overlap between true predictions than between false predictions ( $54 \%$ versus $21 \%$ ). In other words, different programs differed more in the mistakes they made than in their correct predictions.

Principal components analysis (PCA) of the training set (519 promoters and 2,595 non-promoters) was used to give a rough visual representation of the separability of the promoter and non-promoter classes. The first two principal components are plotted in Figure 2a. No one feature was noticeably highly weighted in the first two principal components. Non-promoters form a reasonably tight cluster, while promoters are much more diffuse. This is a consequence of using promoter-centric features. Naively, it would be expected that the

Table 1 Description of features used

\begin{tabular}{ll}
\hline Feature & Information \\
\hline Profisi & DNA melting temperature, as calculated with Fixman \& Freire's method \\
ARTS & Custom SVM kernel using both sequence and structural information \\
N-SCAN & HMM gene predictor - start of $5^{\prime}$ UTR defines TSS \\
FirstEF & Decision tree using $k$-mers, GC and CpG content \\
Eponine & RVM using mixture of Gaussian distributions of position weight matrices \\
ProSOM & Self-organising map trained on base stacking energy \\
EP3 & Base stacking energy \\
Methylation & Experimentally determined CpG methylation profiles \\
Conservation & 17-way vertebrate conservation scores \\
\hline
\end{tabular}

All except methylation and conservation are outputs from prediction programs. ARTS scores were split into + and - strands. Methylation scores were split into stem cell and differentiated categories. 
Table 2 Overlap between whole genome predictions as measured by $\cap / \cup$ (1000 bp tolerance)

\begin{tabular}{lrrrrrrrr}
\hline N-SCAN & 1.000 & & & & & & \\
FirstEF & 0.424 & 1.000 & & & & & \\
Eponine & 0.442 & 0.440 & 1.000 & & & & \\
ProSOM & 0.212 & 0.284 & 0.256 & 1.000 & & & \\
EP3 & 0.209 & 0.317 & 0.244 & 0.575 & 1.000 & & \\
ARTS & 0.308 & 0.314 & 0.319 & 0.161 & 0.162 & 1.000 & \\
Profisi & 0.319 & 0.504 & 0.360 & 0.255 & 0.290 & 0.247 & 1.000 \\
& N-SCAN & FirstEF & Eponine & ProSOM & EP3 & ARTS & Profisi
\end{tabular}

Where more than one prediction existed in a 2,000 bp range, only the prediction with the highest score was counted. For ProSOM, EP3, ARTS, and Profisi, predictions were thresholded to leave $\sim 20,000$ predictions per program.

non-promoter class would be more diffuse, given the many different types of DNA it comprises. The feature weights from these principal components are plotted in Figure 2b. Promoter features appear together, in two groups, while conservation and methylation features are both separate.

Evaluating the contribution of each feature in a support vector machine can be difficult and computationally expensive. We therefore used information gainbased feature ranking to predict the potential contribution of each feature. The predicted ranking is shown in table 5. ARTS [9] was ranked top, which was not surprising given its status as best performer in the last comprehensive prediction review [5]. Surprisingly, the ranking of methylation scores was comparable to many supervised methods. This may be because we only had nonzero scores for $\mathrm{CpG}$ islands, areas associated with promoter activity in general. Conservation was the lowest ranked feature by some distance. This implies that it is only beneficial when combined with other features.

We trained SVMs using both the full set of 11 features, and a reduced set of the 5 top features ranked by information gain. Both were tested on human chromosome 22 only using pppBenchmark 1.3 [5]. The mapped area of chromosome 22 corresponds to $\sim 1 \%$ of the genome. Sensitivity-specificity curves for these tests are shown in Figure 3. The reduced feature set is more

Table 3 Overlap between whole genome predictions as measured by $\mathrm{n} \cup(1000$ bp tolerance), considering only true positive predictions

\begin{tabular}{lrrrrrrrr}
\hline N-SCAN & 1.000 & & & & & & \\
FirstEF & 0.740 & 1.000 & & & & & \\
Eponine & 0.569 & 0.624 & 1.000 & & & & \\
ProSOM & 0.384 & 0.432 & 0.436 & 1.000 & & & \\
EP3 & 0.413 & 0.473 & 0.457 & 0.704 & 1.000 & & \\
ARTS & 0.626 & 0.688 & 0.612 & 0.401 & 0.428 & 1.000 & \\
Profisi & 0.579 & 0.661 & 0.580 & 0.414 & 0.445 & 0.581 & 1.000 \\
& N-SCAN & FirstEF & Eponine & ProSOM & EP3 & ARTS & Profisi \\
& & & & & & & &
\end{tabular}

Table 4 Overlap between whole genome predictions as measured by $\mathrm{N} \cup$ (1000 bp tolerance), considering only false positive predictions

\begin{tabular}{lrrrrrrrr}
\hline N-SCAN & 1.000 & & & & & & \\
FirstEF & 0.218 & 1.000 & & & & & \\
Eponine & 0.294 & 0.315 & 1.000 & & & & \\
ProSOM & 0.109 & 0.210 & 0.164 & 1.000 & & & \\
EP3 & 0.103 & 0.244 & 0.149 & 0.523 & 1.000 & & \\
ARTS & 0.136 & 0.163 & 0.174 & 0.082 & 0.080 & 1.000 & \\
Profisi & 0.168 & 0.416 & 0.241 & 0.195 & 0.232 & 0.131 & 1.000 \\
& N-SCAN & FirstEF & Eponine & ProSOM & EP3 & ARTS & Profisi \\
\hline
\end{tabular}

accurate at high thresholds, while the full set is more accurate at low thresholds. This held true even when we tried a large range of different parameters for both $\mathrm{C}$ (error penalty) and $\gamma$ (Gaussian width). It was decided to combine results from both models for the whole

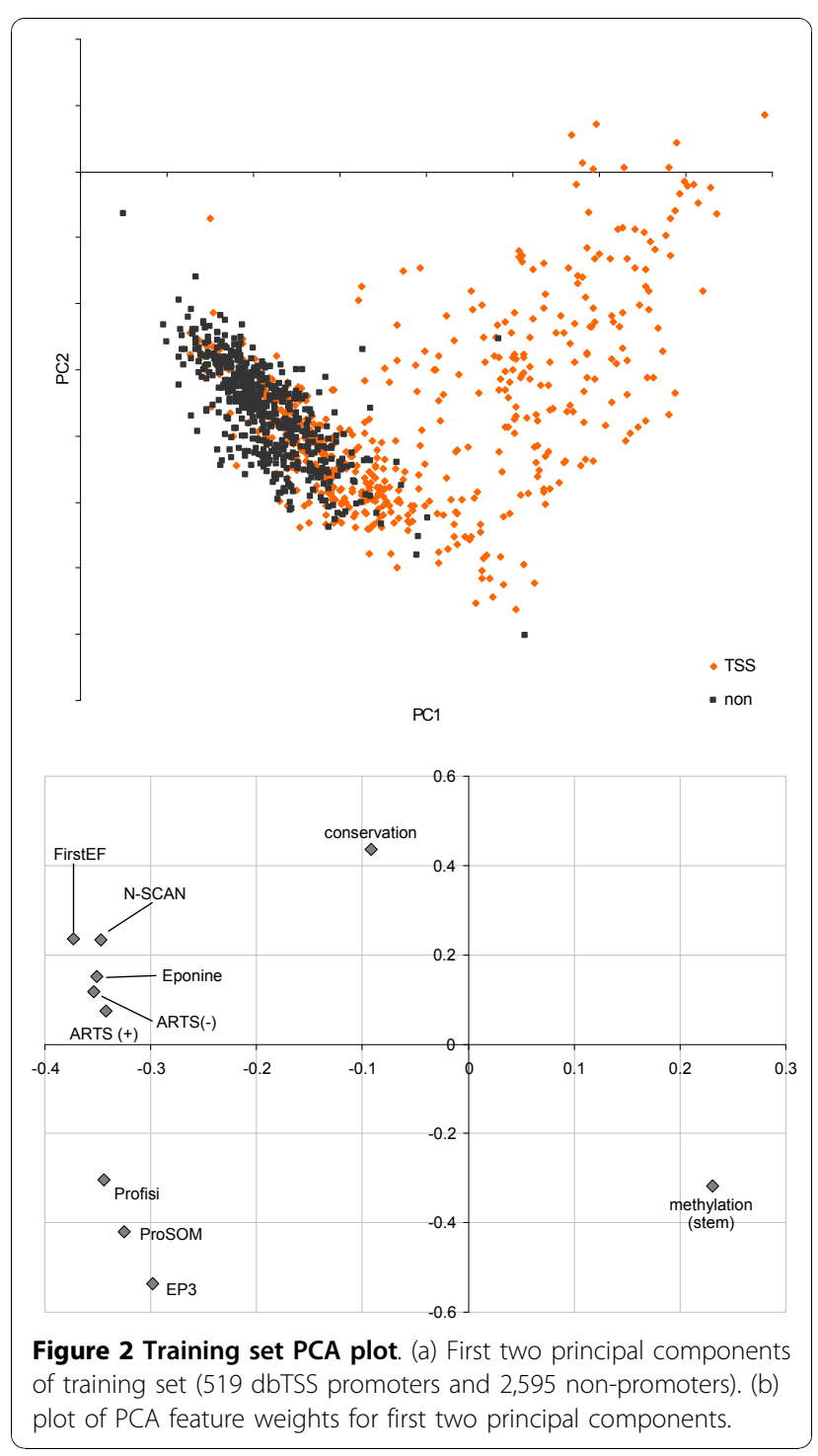


Table 5 Information gain-based ranking of features based on analysis of the training set with Weka 3.6 using default parameters

\begin{tabular}{ll}
\hline Score & Feature \\
\hline 0.196 & ARTS (+ strand) \\
0.195 & ARTS (- strand) \\
0.182 & Profisi \\
0.161 & FirstEF \\
0.157 & N-SCAN \\
0.153 & Methylation (differentiated cells) \\
0.150 & Methylation (stem cells) \\
0.113 & Eponine \\
0.105 & ProSOM \\
0.090 & EP3 \\
0.031 & 17-way conservation \\
\hline
\end{tabular}

The top 5 features in this ranking were used to train the 'high specificity' SVM.

genome test. We used the reduced model unless its predicted probability fell below 0.94 , in which case the full model was used instead. This idea of "punting" switching classifiers when the score falls below a certain threshold - has been successfully used in protein family prediction [29]. To verify the soundness of the idea, we reran pppBenchmark with the combined predictions. The area under the resulting curve was the same as the area under the union of the two previous results. Having learned our threshold on $1 \%$ of the data, we then calculated predictions for the whole genome.

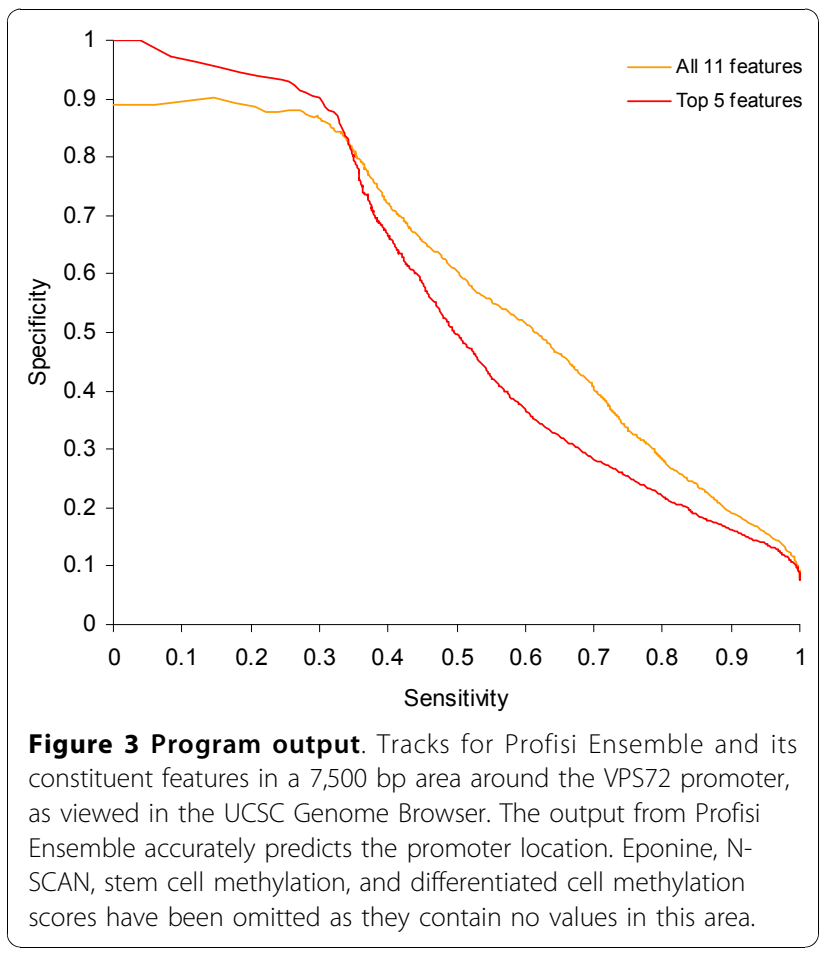

An example of one of the final predictions is given in Figure 4. Shown is the area $3 \mathrm{kbp}$ around the VPS72 promoter, a promoter not associated with a CpG island. Plots of features with nonzero values in this area, as well as GC content, are shown beneath the main prediction. The peak in the Profisi Ensemble score coincides well with both the RefSeq and CAGE annotations. In addition, scores either side of the peak are very low, indicating the reduction in noise achieved by the ensemble approach, compared to individual predictors.

Whole genome predictions were evaluated using pppBenchmark 1.3. pppBenchmark evaluates predictions versus cap analysis of gene expression (CAGE) and RefSeq annotations, using both binning and distance-based protocols, for an accurate overall view of predictive power. The best performer in the original benchmarking was ARTS.

Figure 5 shows sensitivity-specificity curves for a number of programs using pppBenchmark's protocol 2A (considered the most important score by pppBenchmark's authors). Profisi Ensemble shows the best performance over the vast majority of the curve. Curves for $\mathrm{CpG}$ and non-CpG performance are given in Additional file 1.

Profisi Ensemble's pppBenchmark performance was as follows: 1A: 0.224 1B: 0.407 2A: 0.520 2B: 0.691 . This is an improvement over ARTS in all categories. The PPP score is defined as the harmonic mean of the four scores given above. Profisi Ensemble's PPP score was 0.389, or $14 \%$ better than ARTS. Figure 6 shows these 2A and PPP scores. In summary, Profisi Ensemble is currently the most accurate predictor of human promoter activity.

We also performed comparisons with MetaProm and EnsemPro. As we did not have access to predictions from these programs, we evaluated Profisi Ensemble using their evaluation rules.

MetaProm uses a combination of dbTSS and RefSeq as its evaluation set, taking not only a single representative TSS from dbTSS, but also the most upstream and downstream, to evaluate performance in the prediction of alternative TSS. Predictions within $2 \mathrm{kbp}$ of the TSS were considered valid. The results of our evaluation are shown in Figure 7a. MetaProm performed better at high specificities, while Profisi Ensemble performed better at high sensitivities.

EnsemPro uses the EPD as its reference set. As mentioned above, the EPD is not considered a representative set of human promoters. Only an area $1.5 \mathrm{kbp}$ in size around the TSS was examined. Predictions within $200 \mathrm{bp}$ (upstream) or $100 \mathrm{bp}$ (downstream) were counted as true positives. The results of the evaluation are shown in Figure 7b. Profisi Ensemble shows roughly equivalent performance to EnsemPro in this evaluation, although results may not be exact due to variations in the dataset (see Methods). 


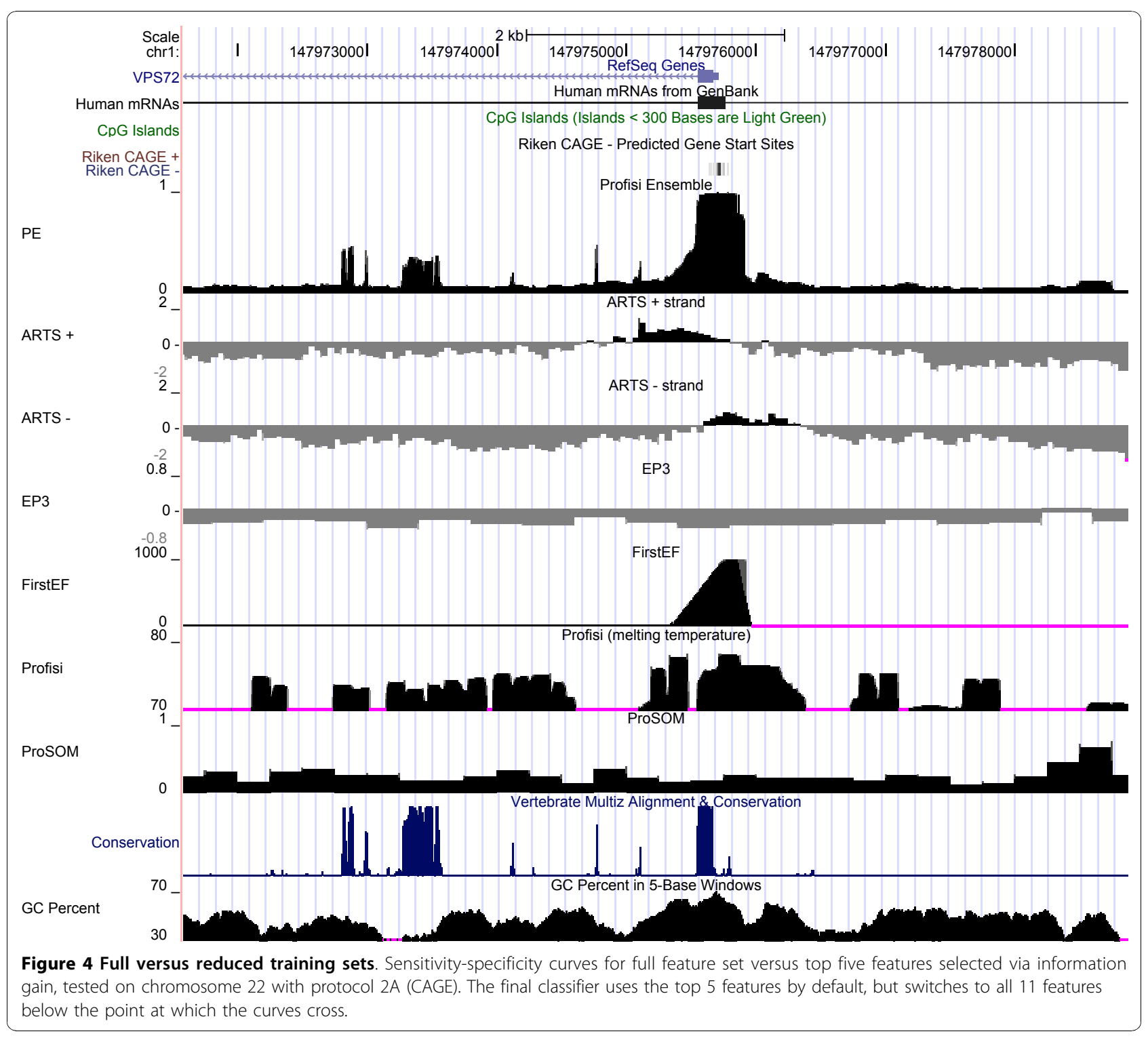

\section{Conclusions}

Profisi Ensemble uses a two layer approach to prediction. Two SVMs are trained using scores from existing prediction programs as features. The predictions from these SVMs are then combined in an either/or manner.

In this work, we have demonstrated the substantial heterogeneity of promoter predictions from current methods. We showed that this heterogeneity enables performance improvements via an ensemble approach. Finally, we have shown that high-sensitivity and highspecificity classifiers may be combined using a "punting" approach to guarantee higher performance across a range of thresholds.

In many fields, diverse predictors for the same task exist, often of broadly similar performance. If these predictors are sufficiently heterogenous, there is merit in exploring an ensemble-based approach. If high specificity/precision is required, consideration should be given to using feature ranking to ensure that only useful features are included.

The same technique we have used for human predictions could be extended to any other genome, as long as sufficiently diverse predictions are available for it. Detailed instructions on applying our method to other organisms are included in Additional file 2. Many prediction programs are able to output predictions for multiple genomes. EP3, for example contains models for ten model organisms [25]. As we have used a supervised approach, a high quality training set (preferably based on experimental data, like the dbTSS) is essential, however. 


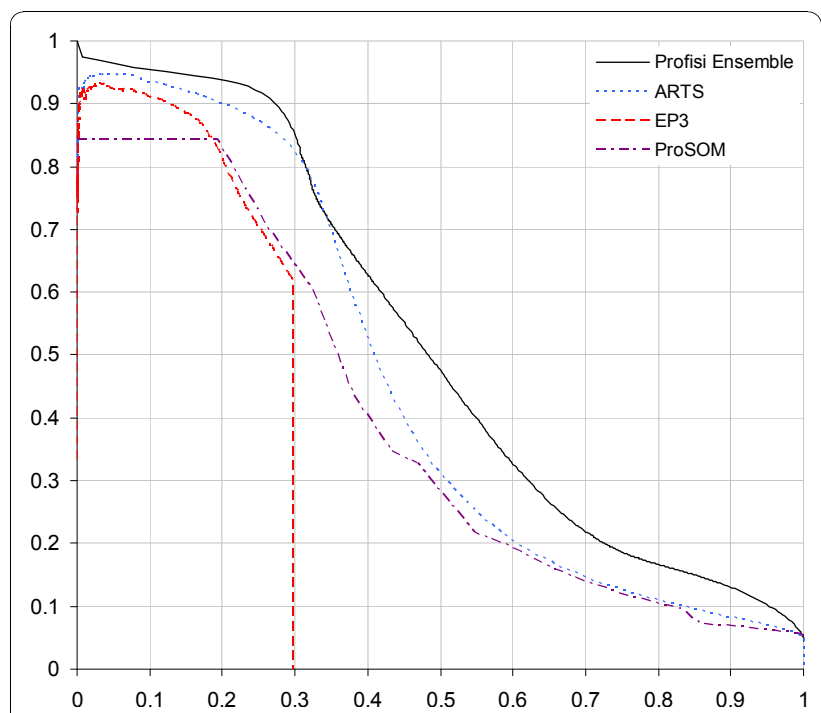

Figure 5 Performance comparison (sensitivity-specificity scores). Sensitivity-specificity curves for various promoter prediction programs, tested on human genome build hg 18 with protocol 2A (CAGE).

5 bp resolution probability scores for genome builds hg17 and hg18 are available from http://mlg.ucd.ie/ profisiensemble. 1 bp resolution scores are available on request. Source code is available in Additional file 3.

\section{Methods}

To assess the overlap between predictions from different programs, whole genome predictions were downloaded from the UCSC Genome Browser [30] and from the websites associated with the programs. Where multiple predictions existed around a single locus (2000 base pairs), only the prediction with the highest score was kept. Programs giving discrete predictions (N-SCAN [28],

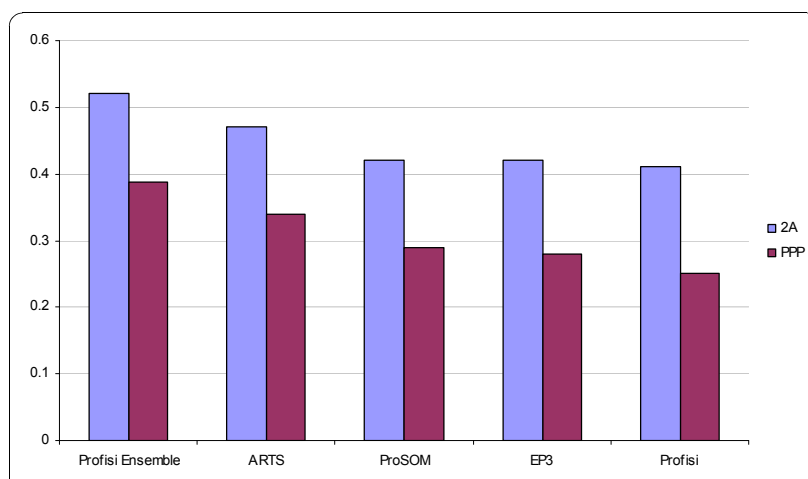

Figure 6 Overall performance as evaluated by pppBenchmark. Protocol 2A represents distance-based performance on CAGE tags, while PPP is the harmonic mean of four separate measurements using both CAGE and gene annotation, and represents overall predictive power. Scores for ARTS, ProSOM, and EP3 were taken from the original pppBenchmark evaluation.

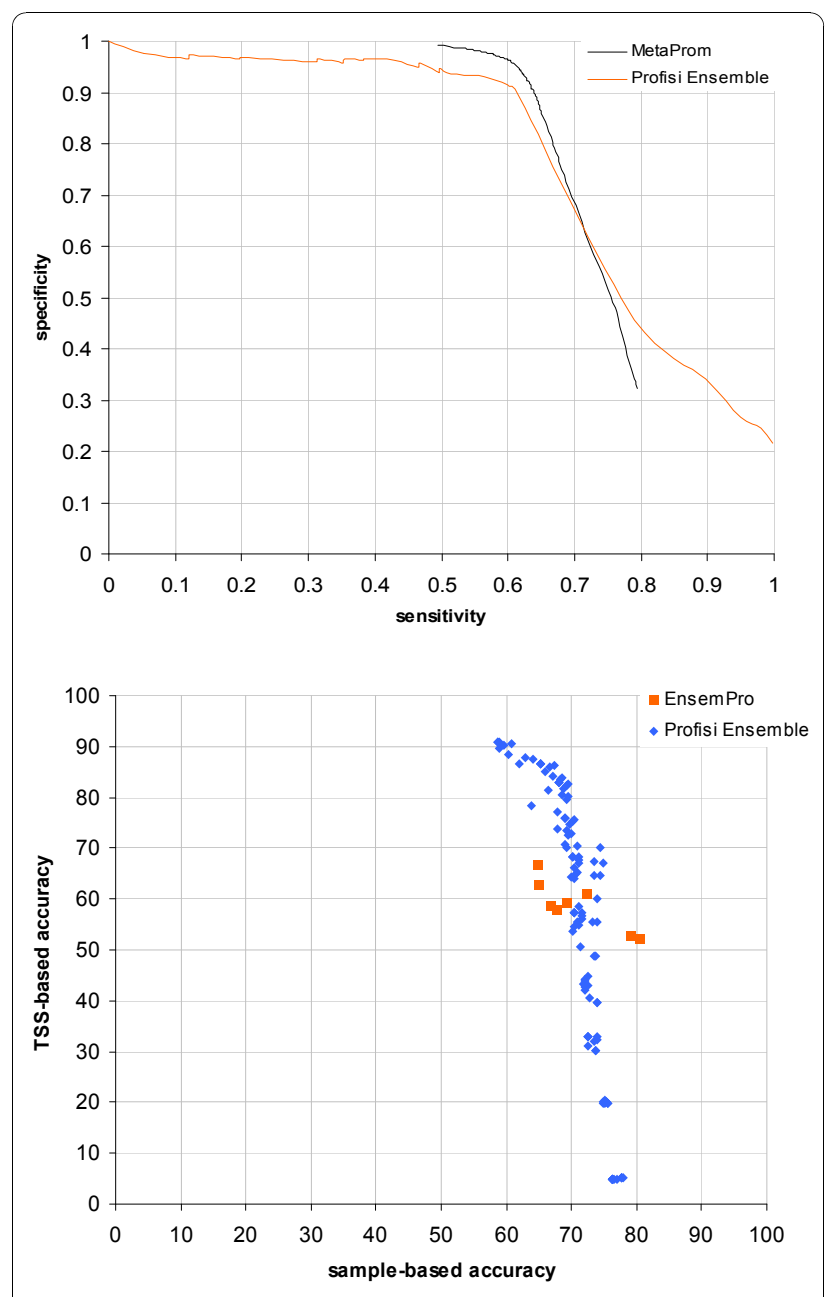

Figure 7 Comparison with other ensemble methods. (a) Evaluation of Profisi Ensemble and MetaProm, using 42,536 TSS drawn from dbTSS and refSeq, in the areas covered by the dbTSS annotation, with a tolerance of 2,000 bp. (b) Evaluation of Profisi Ensemble and EnsemPro (weighted average voting), in random 1.5 kbp areas around Eukaryotic Promoter Database start sites, with a tolerance of $200 \mathrm{bp}$ upstream and $100 \mathrm{bp}$ downstream. EnsemPro scores represent the average of multiple runs.

FirstEF [26], and Eponine [27]) had roughly 20,000 predictions each. The remaining programs gave continuous scores for the whole genome. These scores were thresholded to also leave $\sim 20,000$ predictions per program. Overlap between sets was measured by dividing set intersection by set union for each pair of programs. Overlap was measured for (a) all predictions, (b) true positive predictions only, and (c) false positive predictions only. Predictions within 1,000 bp of the 5' end of a RefSeq first exon were counted as true positives.

$\mathrm{N}$-SCAN, FirstEF, and Eponine predictions were downloaded from the UCSC Genome Browser. These point predictions were converted to continuous scores using a 1000 base pair window, with the central 200 
base pairs getting the full score, linearly falling to 0 at the edges, giving a trapezoid-type distribution. These parameters were determined using small-scale tests on the ENCODE regions. The remaining features had scores for all base pairs. ProSOM and EP3 predictions were obtained using the Java executables available online. ARTS predictions were download from the ARTS website. Profisi melting temperatures were downloaded from the human genome melting map. Methylation scores were obtained from a whole-genome methylation map of 15 cell lines [19] (Island methylation scores from Supplementary Table 1b). Cell lines were split into pluripotent and differentiated categories, and averaged. Scores for the two sperm cell lines were ignored due to the large differences in DNA packing and methylation in these lines. PhastCons 17 -way vertebrate conservation scores were downloaded from the UCSC Genome Browser.

Training examples were drawn from the 44 ENCODE regions which together comprise about $1 \%$ of the human genome. Positive examples were taken from the dbTSS [31], an experimentally verified database which is already used as the training set for [9] and [24]. There were 519 TSS from the database in the ENCODE regions. Five times as many negative examples were selected, to account for the greater variety of negative examples (intergenic, exons, introns, non-promoter regulation such as enhancers, insulators, etc.). These negative examples were all at least 1000 base pairs from the nearest TSS.

Principal components analysis was performed in Weka 3.6 [32] using the default parameters, giving five principal components. Information gain-based feature selection was also performed in Weka using the default parameters.

LibSVM 2.9 [33] was used to train the models and generate predictions, due to its speed, stability, and availability for multiple platforms. It is not multithreaded, but was easily parallelizable as each chromosome was a separate test file. The default kernel - the radial basis function (RBF) was used. Weights were used to compensate for the uneven class sizes. Features were normalized in the range 0-1 to maximize sparsity. LibSVM was set to output a probability rather than a margin score. The error penalty $(\mathrm{C})$ and the tightness parameter $(\gamma)$ were chosen using the supplied grid.py.

Figure 3 shows performance on the mapped portions of human chromosome 22 ( $1 \%$ of the genome). The reduced set outperforms the full set above a certain crossover point. The probability from the reduced set at this crossover was 0.94 . To ensure that this was not due to the SVM parameters resulting in optimization of different areas of the curve, a wide range of values of $\mathrm{C}$ and $\gamma$ were tried. In all cases, the area under the curve was reduced, but the shape of the curve stayed the same. Based on this, we decided to combine predictions from both with an either/or approach. Reduced model predictions below 0.94 were discarded and replaced with predictions from the full set, which were scaled so that the highest value remaining was 0.94 .

As the evaluations for both MetaProm and EnsemPro are based on the older system of point predictions, the continuous scores from Profisi Ensemble had to also be converted to point predictions. We did this using a combination of thresholding and clustering. Thresholding meant throwing away all predictions below a certain level. Clustering meant finding the location with the highest score, and discarding all locations within $n$ base pairs of it, then finding the location with the next highest score and doing the same, etc., until the last location was reached. For both the MetaProm and EnsemPro evaluations, we performed a grid search on the thresholding and clustering parameters, and kept the best performing ones. Cluster sizes were 50-2000 for EnsemPro and 500 for MetaProm. Thresholds were 0-1 for both. Predictions in areas not examined by MetaProm and EnsemPro were discarded.

42,536 TSS in 14,566 sequences were obtained from the MetaProm authors, along with sensitivity-specificity curves. MetaProm $\mathrm{CpG}$ and non-CpG scores were combined.

The EnsemPro evaluation describes discarding EPD TSS where there missing bases within $1,150 \mathrm{bp}$ of the TSS, leaving 400 TSS from 1871. As we were unable to find any missing bases, we used all TSS. EnsemPro figures were obtained from Table 2 of the EnsemPro paper. Weighted majority voting figures were used, as this was the best performing method.

Predictions were made for genome build hg17, and reduced to 5 base pair resolution and converted to build hg18 for testing with pppBenchmark.

\section{Additional material}

Additional file 1: pppBenchmark CAGE evaluation. Evaluation of Profisi Ensemble versus ARTS, EP3, and ProSOM using pppBenchmark protocol 2A, for all predictions, CpG predictions, and non-CpG predictions.

Additional file 2: Instructions for other organisms. Instructions for implementing the Profisi Ensemble method for organisms other than human

Additional file 3: Profisi Ensemble source. Java source code for routines used to preprocess data and process results

\section{Acknowledgements}

This work was funded by Science Foundation Ireland (SFI) grants 08/SRC/ 11407 and 07/IN.1/B1783. The authors would like to thank Andreas Wilm and Junwen Wang for his help. 


\section{Author details}

${ }^{1}$ Complex and Adaptive Systems Laboratory (CASL), University College Dublin, Belfield, Dublin 4, Ireland. ${ }^{2}$ The Conway Institute of Biomolecular and Biomedical Research, University College Dublin, Belfield, Dublin 4, Ireland. ${ }^{3}$ BRF, Center for Biotechnology (CeBiTec), Bielefeld University, Bielefeld, Germany.

\section{Authors' contributions}

$\mathrm{DH}$ and PC suggested the project and edited the manuscript. DD designed and performed the analysis, created and evaluated the predictions, and drafted the manuscript. MS performed PCA analysis. All authors read and approved the final manuscript.

Received: 21 April 2010 Accepted: 30 November 2010 Published: 30 November 2010

\section{References}

1. The FANTOM Consortium, Carninci P, Kasukawa T, et al: The Transcriptional Landscape of the Mammalian Genome. Science 2005, 309:1559-1563.

2. Cheng J, Kapranov P, Drenkow J, Dike S, Brubaker S, Patel S, Long J, Stern D, Tammana H, Helt G, Sementchenko V, Piccolboni A, Bekiranov S, Bailey DK, Ganesh M, Ghosh S, Bell I, Gerhard DS, Gingeras TR: Transcriptional Maps of 10 Human Chromosomes at 5-Nucleotide Resolution. Science 2005, 308:1149-1154.

3. Kapranov P, Cheng J, Dike S, Nix DA, Duttagupta R, Willingham AT, Stadler PF, Hertel J, Hackermuller J, Hofacker IL, Bell I, Cheung E, Drenkow J, Dumais E, Patel S, Helt G, Ganesh M, Ghosh S, Piccolboni A, Sementchenko V, Tammana H, Gingeras TR: RNA Maps Reveal New RNA Classes and a Possible Function for Pervasive Transcription. Science 2007, 316:1484-1488.

4. Carninci P, Sandelin A, Lenhard B, Katayama S, Shimokawa K, Ponjavic J, Semple CAM, Taylor MS, Engstrom PG, Frith MC, Forrest ARR, Alkema WB, Tan SL, Plessy C, Kodzius R, Ravasi T, Kasukawa T, Fukuda S, KanamoriKatayama M, Kitazume Y, Kawaji H, Kai C, Nakamura M, Konno H, Nakano K, Mottagui-Tabar S, Arner P, Chesi A, Gustincich S, Persichetti F, Suzuki H, Grimmond SM, Wells CA, Orlando V, Wahlestedt C, Liu ET, Harbers M, Kawai J, Bajic VB, Hume DA, Hayashizaki Y: Genome-wide analysis of mammalian promoter architecture and evolution. Nat Genet 2006, 38:626-635.

5. Abeel T, Van de Peer Y, Saeys Y: Toward a gold standard for promoter prediction evaluation. Bioinformatics 2009, 25:i313-320

6. Saxonov S, Berg P, Brutlag DL: A genome-wide analysis of CpG dinucleotides in the human genome distinguishes two distinct classes of promoters. Proceedings of the National Academy of Sciences of the United States of America 2006, 103:1412-1417.

7. Roider HG, Lenhard B, Kanhere A, Haas SA, Vingron M: CpG-depleted promoters harbor tissue-specific transcription factor binding signalsimplications for motif overrepresentation analyses. Nucl Acids Res 2009, 37:6305-6315

8. Goni JR, Perez A, Torrents D, Orozco M: Determining promoter location based on DNA structure first-principles calculations. Genome Biology 2007, 8:R263.

9. Sonnenburg S, Zien A, Ratsch G: ARTS: accurate recognition of transcription starts in human. Bioinformatics 2006, 22:e472-480.

10. Dineen DG, Wilm A, Cunningham P, Higgins DG: High DNA melting temperature predicts transcription start site location in human and mouse. Nucl Acids Res 2009, gkp821.

11. Wang J, Ungar L, Tseng H, Hannenhalli S: MetaProm: a neural network based meta-predictor for alternative human promoter prediction. $B M C$ Genomics 2007, 8:374.

12. Won H, Kim M, Kim S, Kim J: EnsemPro: An ensemble approach to predicting transcription start sites in human genomic DNA sequences. Genomics 2008, 91:259-266.

13. Wan J, Kang S, Tang C, Yan J, Ren Y, Liu J, Gao X, Banerjee A, Ellis LBM, Li T: Meta-prediction of phosphorylation sites with weighted voting and restricted grid search parameter selection. Nucl Acids Res 2008, gkm848.

14. Boulesteix A, Porzelius C, Daumer M: Microarray-based classification and clinical predictors: on combined classifiers and additional predictive value. Bioinformatics 2008, 24:1698-1706.
15. Kedarisetti KD, Kurgan L, Dick S: Classifier ensembles for protein structural class prediction with varying homology. Biochemical and Biophysical Research Communications 2006, 348:981-988.

16. Polikar R: Ensemble Based Systems in Decision Making. IEEE Circuits and Systems Magazine 2006, 6:45-21.

17. Zenko $B^{v}$ : Is Combining Classifiers Better than Selecting the Best One. MACHINE LEARNING 2004, 54:255-273.

18. Bajic VB, Brent MR, Brown RH, Frankish A, Harrow J, Ohler U, Solovyev W, Tan SL: Performance assessment of promoter predictions on ENCODE regions in the EGASP experiment. Genome Biol 2006, 7:S3.

19. Straussman R, Nejman D, Roberts D, Steinfeld I, Blum B, Benvenisty N, Simon I, Yakhini Z, Cedar H: Developmental programming of CpG island methylation profiles in the human genome. Nat Struct Mol Biol 2009, 16:564-571.

20. Aloui A, Tagourti J, El May A, Joseleau Petit D, Landoulsi A: The effect of methylation on some biological parameters in Salmonella enterica serovar Typhimurium. Pathologie Biologie, Corrected Proof.

21. Doerfler W: The Significance of DNA Methylation Patterns: Promoter Inhibition by Sequence-Specific Methylation is One Functional Consequence. Philosophical Transactions of the Royal Society of London. Series B, Biological Sciences 1990, 326:253-265.

22. King DC, Taylor J, Elnitski L, Chiaromonte F, Miller W, Hardison RC: Evaluation of regulatory potential and conservation scores for detecting cis-regulatory modules in aligned mammalian genome sequences. Genome Res 2005, 15:1051-1060.

23. Jin VX, Singer GAC, Agosto-Pérez FJ, Liyanarachchi S, Davuluri RV: Genomewide analysis of core promoter elements from conserved human and mouse orthologous pairs. BMC Bioinformatics 2006, 7:114.

24. Abeel $T$, Saeys $Y$, Rouzé $P$, Van de Peer $Y$ : ProSOM: core promoter prediction based on unsupervised clustering of DNA physical profiles. Bioinformatics 2008, 24:i24-31.

25. Abeel T, Saeys Y, Bonnet E, Rouzé P, Van de Peer Y: Generic eukaryotic core promoter prediction using structural features of DNA. Genome Res 2008, 18:310-23.

26. Davuluri RV, Grosse I, Zhang MQ: Computational identification of promoters and first exons in the human genome. Nat Genet 2001, 29:412-7.

27. Down TA, Hubbard TJP: Computational detection and location of transcription start sites in mammalian genomic DNA. Genome Res 2002, 12:458-61.

28. Gross SS, Brent MR: Using multiple alignments to improve gene prediction. J Comput Biol 2006, 13:379-93.

29. Melvin I, Weston J, Leslie C, Noble W: Combining classifiers for improved classification of proteins from sequence or structure. BMC Bioinformatics 2008, 9:389.

30. Kent WJ, Sugnet CW, Furey TS, Roskin KM, Pringle TH, Zahler AM, Haussler D: The human genome browser at UCSC. Genome Res 2002, 12:996-1006

31. Suzuki Y, Yamashita R, Nakai K, Sugano S: DBTSS: DataBase of human Transcriptional Start Sites and full-length cDNAs. Nucl Acids Res 2002, 30:328-331.

32. Hall M, Frank E, Holmes G, Pfahringer B, Reutemann P, Witten IH: The WEKA data mining software: an update. SIGKDD Explor News/ 2009, 11:10-18.

33. Chang Chih-chung, Lin Chih-jen: LIBSVM: a library for support vector machines. 2001.

doi:10.1186/1471-2164-11-677

Cite this article as: Dineen et al:: Ensemble approach combining multiple methods improves human transcription start site prediction. BMC Genomics 2010 11:677. 